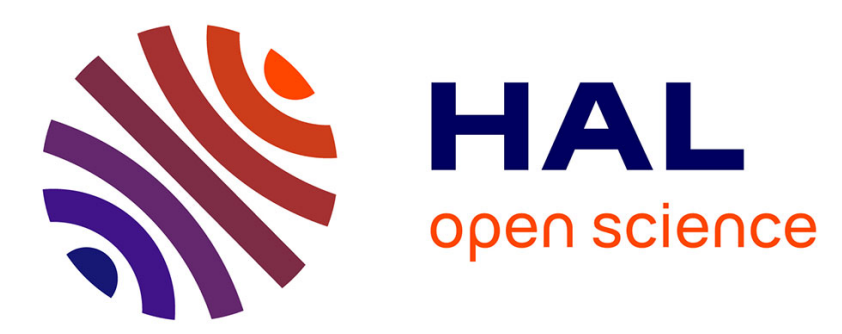

\title{
Short-time evolution of Lagrangian velocity gradient correlations in isotropic turbulence
}

\author{
Le Fang, Wouter J.T. Bos, Guodong Jin
}

\section{To cite this version:}

Le Fang, Wouter J.T. Bos, Guodong Jin. Short-time evolution of Lagrangian velocity gradient correlations in isotropic turbulence. Physics of Fluids, 2015, 27 (12), pp.125102. 10.1063/1.4936140 . hal-01298331

\section{HAL Id: hal-01298331 \\ https://hal.science/hal-01298331}

Submitted on 6 Apr 2016

HAL is a multi-disciplinary open access archive for the deposit and dissemination of scientific research documents, whether they are published or not. The documents may come from teaching and research institutions in France or abroad, or from public or private research centers.
L'archive ouverte pluridisciplinaire HAL, est destinée au dépôt et à la diffusion de documents scientifiques de niveau recherche, publiés ou non, émanant des établissements d'enseignement et de recherche français ou étrangers, des laboratoires publics ou privés. 


\title{
Short-time evolution of Lagrangian velocity gradient correlations in isotropic turbulence
}

\author{
L. Fang, ${ }^{1}$ W. J. T. Bos, ${ }^{2}$ and G. D. Jin ${ }^{3, a)}$ \\ ${ }^{1}$ LMP, Ecole Centrale de Pékin, Beijing University of Aeronautics and Astronautics, \\ Beijing 100191, China \\ ${ }^{2}$ LMFA, CNRS, Ecole Centrale de Lyon-Université de Lyon, 69130 Ecully, France \\ ${ }^{3}$ LNM, Institute of Mechanics, Chinese Academy of Sciences, Beijing 100190, China
}

(Received 10 April 2015; accepted 6 November 2015; published online 9 December 2015)

\begin{abstract}
We show by direct numerical simulation (DNS) that the Lagrangian cross correlation of velocity gradients in homogeneous isotropic turbulence increases at short times, whereas its auto-correlation decreases. Kinematic considerations allow to show that two invariants of the turbulent velocity field determine the short-time velocity gradient correlations. In order to get a more intuitive understanding of the dynamics for longer times, heuristic models are proposed involving the combined action of local shear and rotation. These models quantitatively reproduce the effects and disentangle the different physical mechanisms leading to the observations in the DNS. () 2015 AIP Publishing LLC. [http://dx.doi.org/10.1063/1.4936140]
\end{abstract}

\section{INTRODUCTION}

The study of the Lagrangian dynamics of the velocity gradient tensor in turbulent flows has recently received a considerable amount of attention. Its investigation helps us to understand important phenomena involving the small scales of turbulent flows, such as the preferential vorticity alignment, the skewness of longitudinal velocity gradients, small scale intermittency, etc. ${ }^{1}$ Recent developments in experimental techniques allow nowadays to deterministically track the development of the velocity gradient tensor in Eulerian ${ }^{2}$ and Lagrangian settings. ${ }^{3}$ These results, combined with results from Direct Numerical Simulations (DNS) (e.g., Refs. 4 and 5) provide unprecedented possibilities to understand the origin of these interesting phenomena.

In order to understand the phenomenology of the velocity gradient evolution, simplified models are needed which allow to identify the different physical features. One of the simplest models is the so-called restricted Euler (RE) equation for the velocity gradient tensor. This equation is obtained by removing the viscous diffusion term and the anisotropic part of the pressure Hessian from the evolution equation of the velocity-gradient tensor, only leaving the advection term, the self-stretching term, and the symmetric part of the pressure Hessian. ${ }^{6}$ There are many studies on the properties of this RE system, investigating, in particular, the evolution of the invariants and their probability density functions. ${ }^{6-9}$ However, the system evolves to a singular state, ${ }^{10}$ so that the time-evolution at long times is not comparable to realistic Navier-Stokes dynamics. In particular, the influence of the anisotropic part of the pressure Hessian on the distribution of tensor invariants seems to be significant. ${ }^{11,12}$ More recent theoretical approaches attempt to model these remaining terms using geometrical considerations, ${ }^{13,14}$ assumptions on the short-time deformation, ${ }^{15}$ or assuming Gaussianity of the pressure Hessian. ${ }^{16}$ These approaches have, for instance, allowed to better understand the Lagrangian evolution of the time-correlations of vorticity alignment. ${ }^{17,18}$ The other term omitted from the restricted Euler model, i.e., the viscous term, acts as a damping and greatly influences the Lagrangian time evolution of the velocity gradient tensor. A popular model to represent the effects of the viscous damping is the linear damping model,${ }^{19}$ which is formally quite

\footnotetext{
a) Author to whom correspondence should be addressed. Electronic mail: gdjin@lnm.imech.ac.cn
} 
simple, but can be regarded as a good approximation for a number of applications. ${ }^{1,20}$ This is not so at short times, as will be shown in the present investigation.

Whereas, as mentioned above, the evolution of the invariants and their probability density distribution have received a large amount of interest, the Lagrangian time correlations have not been investigated so much. One recent study on the subject is the work by Yu and Meneveau. 21,22 A tensor-based correlation function was defined to represent the time evolution property along the trajectory of a fluid particle. It was shown that this correlation function always decreases, and the correlation-time is related to the local Kolmogorov time scale. In the present contribution, we will show that a component of this correlation function, the cross correlation, does not always decrease. Instead, it non-monotonically varies with the time lag, i.e., it initially increases for a couple of Kolmogorov time scales, before it starts to decrease. In order to better understand the short-time evolution of the Lagrangian velocity-gradient correlations, we combine DNS, kinematics, and heuristic modeling.

In Section II, we will define the different components of the Lagrangian velocity gradient correlation tensor and we will show that DNS results predict an increase of one of the components of this tensor. In Section III, it is rigorously shown that at short times the pressure Hessian can cause this effect, neglecting other contributions to the evolution. A simplified kinematic model will be proposed to explain this interesting phenomenon in Section IV and the discussion and conclusion are presented in Section V.

\section{BEHAVIOUR OF THE LAGRANGIAN VELOCITY GRADIENT CORRELATIONS IN DIRECT NUMERICAL SIMULATION}

\section{A. The Lagrangian velocity gradient tensor}

We consider isotropic incompressible turbulence. The Lagrangian velocity gradient, denoted by

$$
A_{i j}\left(\boldsymbol{x}, t_{0} \mid t\right),
$$

is the value of $\partial_{j} u_{i}$ evaluated at time $t$ at the position of the fluid particle which passed through $\boldsymbol{x}$ at time $t_{0}$. In the present paper, we only consider the case of $t>t_{0}$. The notation $\partial_{i}$ is an abbreviation for the partial derivative $\partial / \partial x_{i}$. We note that in general, for $t \neq t_{0}$,

$$
A_{i j}\left(\boldsymbol{x}, t_{0} \mid t\right) \neq \partial_{j}\left(u_{i}\left(\boldsymbol{x}, t_{0} \mid t\right)\right) .
$$

In particular, $A_{i i}\left(\boldsymbol{x}, t_{0} \mid t\right)=0$ by incompressibility, and this does not hold for $\partial_{i} u_{i}\left(\boldsymbol{x}, t_{0} \mid t\right)$. Inequality (2) complicates the analysis of second-order correlations of the Lagrangian velocity gradient tensor, since we cannot link them directly to the velocity-correlations. For brevity, we will denote $A_{i j}\left(\boldsymbol{x}, t_{0} \mid t\right)$ by $A_{i j}(t)$. Without losing generality, we also define $t_{0}=0$ in Secs. III-V.

The Lagrangian evolution of $A_{i j}$ is given by

$$
\dot{A}_{i j}=-A_{p j} A_{i p}-P_{i j}+v \partial_{p}^{2} A_{i j},
$$

with $P_{i j}=\partial_{i} \partial_{j} p$ the pressure Hessian and $v$ the viscosity.

We further introduce the correlation

$$
B_{i j m n}(t)=\left\langle A_{i j}(0) A_{m n}(t)\right\rangle,
$$

and we omit the parameter $t_{0}=0$. Since we will only consider statistically stationary flows, the temporal correlations depend only on the time lag $t-t_{0}=t$.

At $t=t_{0}$, elementary tensorial kinematics show that, due to isotropy, homogeneity, and incompressibility,

$$
B_{i j m n}(0)=\frac{2}{15}\left(\delta_{i m} \delta_{j n}-\frac{1}{4}\left(\delta_{i j} \delta_{m n}+\delta_{i n} \delta_{j m}\right)\right) \frac{\epsilon}{v},
$$

where the dissipation rate $\epsilon$ is related to the velocity gradients by

$$
\epsilon=15 v\left\langle\left(\frac{\partial u_{1}}{\partial x_{1}}\right)^{2}\right\rangle .
$$


All components of $B_{i j m n}\left(t_{0}\right)$ are thus determined by one scalar invariant, the dissipation. In the following, we will consider the time dependence of $B_{i j m n}(t)$, and, in particular, the transverse autoand cross correlations, $B_{1212}$ and $B_{1221}$. Their values at $t=t_{0}$ are

$$
B_{1212}(0)=\frac{2}{15} \frac{\epsilon}{v}, \quad B_{1221}(0)=-\frac{1}{30} \frac{\epsilon}{v} .
$$

At later times, we will evaluate the different correlations as compared to their value at $t=t_{0}$. For instance,

$$
\tilde{B}_{1212}(t) \equiv \frac{B_{1212}(t)}{B_{1212}(0)}
$$

Intuitively we would expect the norm of these correlations to decay in time, since dynamics on trajectories have a finite time-correlation. It is observed that at short times this is the case for $\tilde{B}_{1212}(t)$, but it is not so for $\tilde{B}_{1221}(t)$.

\section{B. Numerical observations}

A pseudo-spectral method is used to solve the Navier-Stokes equations in a periodically cubic box of size $2 \pi$. A large-scale random forcing scheme is added to the Navier-Stokes equations to produce and maintain statistically stationary isotropic turbulent flows. The details of the calculation can be found in Refs. 23 and 24. The effect of external forcing was discussed by Jeong and Girimaji. ${ }^{25}$ The isotropic turbulent flows at four Taylor's microscale Reynolds numbers, $R e_{\lambda}=74,101$, 205, and 433, are simulated to study the effects of the Reynolds number on the cross correlation of velocity gradients. The flow parameters in different flows are listed in Table I. Here $N$ is the grid resolution in one direction, $u^{\prime}$ is the root mean square of the fluctuation velocity, $L_{f}$ is the integral length scale of the flow, $v_{K}=(v \epsilon)^{1 / 4}$ is the Kolmogorov velocity scale, and $\tau_{K}=\langle v / \epsilon\rangle^{1 / 2}$ is the Kolmogorov time scale. The first three cases are generated using our in-house code and the last one is obtained using the public turbulence database at the Johns Hopkins University. ${ }^{26}$ When the turbulent flow field reaches a statistically stationary state, the initial positions of $4 \times 10^{5}$ fluid particles are recorded and the trajectories of these particles are then advanced in time using a fourth-order Adams-Moulton method according to $d \mathbf{x} / d t=\mathbf{u}(\mathbf{x}(t), t)$, where $\mathbf{u}(\mathbf{x}(t), t)$ is the fluid velocity experienced by one of the fluid particles, obtained from the Eulerian fluid velocity field using a 6th-order Lagrangian interpolation. ${ }^{27}$ The Lagrangian velocity gradient is calculated along the trajectory of a fluid particle. First, we compute the velocity gradient field in an Eulerian frame, then, we obtain the Lagrangian velocity gradient experienced by a fluid particle also using a 6th-order Lagrangian interpolation. For single-point two-time correlations of the velocity gradient, we obtain the velocity gradient at different times at the fixed initial positions of the $4 \times 10^{5}$ fluid particles. As the Lagrangian correlation of velocity gradient decays more slowly than the single-point two-time correlation of the velocity gradient, we calculate the correlations for about $30 \tau_{K}$ so that the Lagrangian correlation of velocity gradient decays to zero. The number of fluid particles used for the determination of the Lagrangian correlation is sufficient to ensure converged, smooth correlation functions, as shown in Fig. 1.

In Fig. 1 it is shown that the correlation $\tilde{B}_{1212}$ decays monotonically, but this is not so for $\tilde{B}_{1221}$. This last quantity increases to a value of around 1.5 after a couple of Kolmogorov times. After that time it starts to decay. At long times this correlation should go to zero, as do the other ones,

TABLE I. Parameters of the considered flows.

\begin{tabular}{lrrcccccc}
\hline \hline Case & $N^{3}$ & $R e_{\lambda}$ & $\epsilon$ & \multicolumn{1}{c}{$v$} & $u^{\prime}$ & $L_{f}$ & $v_{K}$ & $\tau_{K}$ \\
\hline I & $128^{3}$ & 74 & 3434.7 & 0.095 & 19.10 & 0.91 & 4.31 & 0.0051 \\
II & $256^{3}$ & 101 & 3468.0 & 0.049 & 19.52 & 0.99 & 3.62 & 0.0037 \\
III & $512^{3}$ & 205 & 0.2055 & 0.001 & 0.8722 & 3.2283 & 0.1197 & 0.06976 \\
IV & $1024^{3}$ & 433 & 0.0928 & 0.000185 & 0.681 & 1.376 & 0.064 & 0.0446 \\
\hline
\end{tabular}




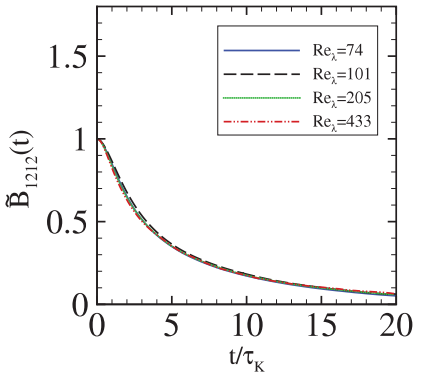

(a)

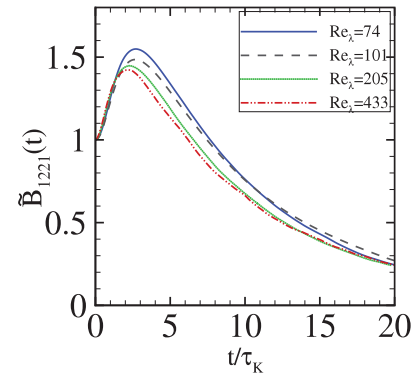

(b)

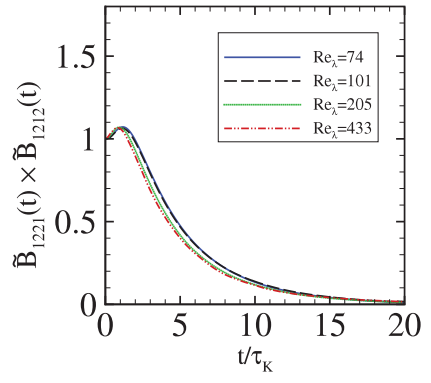

(c)

FIG. 1. The two-time correlation functions calculated in DNS databases. The corresponding values at time $t_{0}=0$ are used for normalization. (a) $\tilde{B}_{1212}(t)$, (b) $\tilde{B}_{1221}(t)$, (c) their product.

reflecting the finite correlation time of a turbulent velocity field. The product of $\tilde{B}_{1221}$ and $\tilde{B}_{1212}$ also increases at short times and decays at long times. The observed tendencies do not seem to be strongly dependent on the Reynolds number. The evolution of the correlations as a function of time in Kolmogorov-units is roughly the same for all Reynolds numbers, but the influence of the Reynolds number on $\tilde{B}_{1221}$ is larger than that on $\tilde{B}_{1212}$.

This short-time increase in $\tilde{B}_{1221}(t)$ is a typically Lagrangian effect, as illustrated in Fig. 2, which shows the comparison between the Lagrangian correlation of $\tilde{B}_{1221}(t)$ and the single-point two-time correlation of $\tilde{B}_{1221}(t)$, where the latter is the correlation of the velocity-gradient calculated at the same position, i.e., without tracking particles. Evidently, the single-point two-time correlation monotonically decays with time, in contrast to the Lagrangian correlation.

In Sec. III, we will try to explain the different behaviours at short times by kinematic considerations.

\section{SHORT-TIME EVOLUTION OF THE CORRELATIONS}

\section{A. Lagrangian correlations}

For short times $\left(t-t_{0}\right)$ we can use the Taylor expansion

$$
A_{i j}^{\text {Taylor }}(t)=A_{i j}\left(t_{0}\right)+\left(t-t_{0}\right) \dot{A}_{i j}\left(t_{0}\right)+\frac{\left(t-t_{0}\right)^{2}}{2} \ddot{A}_{i j}\left(t_{0}\right)+O\left(\left(t-t_{0}\right)^{3}\right) .
$$

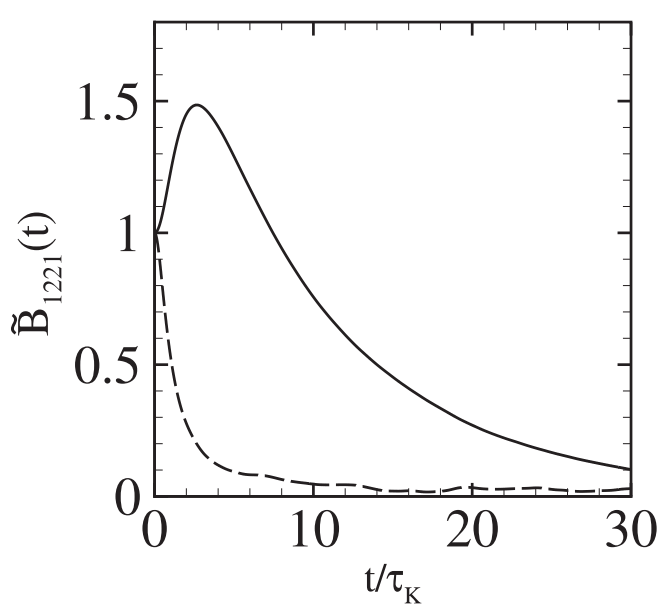

FIG. 2. Comparison between the Lagrangian correlation and the single-point two-time correlation of $\tilde{B}_{1221}(t)$ at Taylor's microscale Reynolds number $R e_{\lambda}=101$, where the solid line denotes the Lagrangian correlation of $\tilde{B}_{1221}(t)$, and the dashed line the single-point two-time correlation of $\tilde{B}_{1221}(t)$. 
The short time evolution of $B_{i j m n}(t)$ can therefore be evaluated by substituting the first nonvanishing contributions of $A_{i j}^{\text {Taylor }}(t)$ in $B_{i j m n}\left(t_{0}, t\right)$. We find, up to second order,

$$
B_{i j m n}(t) \approx B_{i j m n}\left(t_{0}\right)+\left(t-t_{0}\right)\left\langle\dot{A}_{i j}\left(t_{0}\right) A_{m n}\left(t_{0}\right)\right\rangle+\frac{\left(t-t_{0}\right)^{2}}{2}\left\langle\ddot{A}_{i j}\left(t_{0}\right) A_{m n}\left(t_{0}\right)\right\rangle .
$$

We can evaluate the leading-order term, proportional to $\left(t-t_{0}\right)$, by substituting Eq. (3) in Eq. (10). Working out the kinematics for isotropic incompressible turbulence, we find that this term vanishes, reflecting physically the steady-state equilibrium between enstrophy production and dissipation. The vanishing of the first order term can however be shown more directly by considering the time derivative,

$$
\frac{\partial}{\partial t}\left\langle A_{i j}(t) A_{m n}(t)\right\rangle=0
$$

since the flow is statistically stationary. Therefore, we have immediately,

$$
\left\langle\dot{A}_{i j}(t) A_{m n}(t)\right\rangle=-\left\langle A_{i j}(t) \dot{A}_{m n}(t)\right\rangle,
$$

which shows that for $\tilde{B}_{1212}(t)$ and $\tilde{B}_{1221}(t)$, the first-order contribution in Eq. (10) vanishes. In order to explain the short-time behaviour of $\tilde{B}_{1212}(t)$ and $\tilde{B}_{1221}(t)$, we therefore need to retain at least the terms in $\left(t-t_{0}\right)^{2}$.

Using the fact that

$$
\frac{\partial^{2}}{\partial t^{2}}\left\langle A_{i j}(t) A_{m n}(t)\right\rangle=0
$$

we find that

$$
\left\langle\dot{A}_{i j}\left(t_{0}\right) \dot{A}_{m n}\left(t_{0}\right)\right\rangle=-\frac{1}{2}\left(\left\langle\ddot{A}_{i j}\left(t_{0}\right) A_{m n}\left(t_{0}\right)\right\rangle+\left\langle A_{i j}\left(t_{0}\right) \ddot{A}_{m n}\left(t_{0}\right)\right\rangle\right)
$$

leading to

$$
\begin{aligned}
& \left\langle\ddot{A}_{12}\left(t_{0}\right) A_{12}\left(t_{0}\right)\right\rangle=-\left\langle\dot{A}_{12}\left(t_{0}\right) \dot{A}_{12}\left(t_{0}\right)\right\rangle, \\
& \left\langle\ddot{A}_{12}\left(t_{0}\right) A_{21}\left(t_{0}\right)\right\rangle=-\left\langle\dot{A}_{12}\left(t_{0}\right) \dot{A}_{21}\left(t_{0}\right)\right\rangle,
\end{aligned}
$$

which helps us to get rid of the second-order time derivatives. Isotropy allows to express

$$
\left\langle\dot{A}_{i j}\left(t_{0}\right) \dot{A}_{m n}\left(t_{0}\right)\right\rangle=\frac{1}{30}\left(\frac{\epsilon}{v}\right)^{2}\left[(4 F-G) \delta_{i m} \delta_{j n}-(F+G) \delta_{i j} \delta_{m n}+(4 G-F) \delta_{i n} \delta_{j m}\right],
$$

with

$$
F=\left\langle\dot{A}_{i j} \dot{A}_{i j}\right\rangle\left(\frac{v}{\epsilon}\right)^{2}, \quad G=\left\langle\dot{A}_{i j} \dot{A}_{j i}\right\rangle\left(\frac{v}{\epsilon}\right)^{2} .
$$

Using expressions (5), (10), and (16), we find for the normalized correlations,

$$
\begin{aligned}
& \tilde{B}_{1212}(t) \approx 1-\frac{1}{8}\left(\frac{t-t_{0}}{\tau_{K}}\right)^{2}(4 F-G), \\
& \tilde{B}_{1221}(t) \approx 1+\frac{1}{2}\left(\frac{t-t_{0}}{\tau_{K}}\right)^{2}(4 G-F) .
\end{aligned}
$$

This shows that if we can determine the scalar quantities $F$ and $G$, we can determine all the initial trends of the Lagrangian correlation functions. We will first see which constraints are implied by purely kinematic constraints, i.e., without introducing the Navier-Stokes equations. Auto-correlations in stationary turbulence can be assumed to be decaying functions of time, so that, using this constraint on $B_{1111}$ and $B_{1212}$, we have $-F \leq G \leq 4 F$, and $0 \leq F$. This allows but does not demonstrate that $\tilde{B}_{1221}(t)$ is an increasing function in time, since for that, we should have $F / 4 \leq G$. It is normal that at this point we cannot demonstrate this, since we have not used any information on the Navier-Stokes equations, only considerations assuming isotropy and incompressibility. 
Let us at this point add such dynamic information, step by step. For instance, consider that only the viscous term on the RHS of (3) is non-zero. In that case we find, by isotropy that $G=0$, and both correlations in Eqs. (18) and (19) initially decrease. If we now consider a different case, where only the pressure Hessian is non-zero, we find $F=G$ since the pressure Hessian is symmetric in its indices $P_{i j}=P_{j i}$. In that case the cross correlation increases whereas the auto-correlation decreases, as observed in the simulations. This does not prove that it is the pressure Hessian alone which is responsible for the increasing correlation, the self-interaction term (first term on the RHS of Eq. (3)) can also play a role. For that term we have not succeeded to show any simple symmetry properties in a rigorous way. We have therefore proceeded to measure $G / F$ in the direct numerical simulations and we found that $G / F \approx 0.6$ in the simulations, roughly independent of the Reynolds number. This is in the interval $1 / 4 \leq G / F \leq 4$, in which auto-correlations decrease and $\tilde{B}_{1221}(t)$ increases in time.

\section{B. Single-point two-time correlation}

For the case of the single-point two-time correlations of the velocity gradient tensor, we have an additional term on the RHS of the velocity-gradient evolution equation,

$$
\partial_{t} A_{i j}=-u_{m} \partial_{m} A_{i j}-A_{p j} A_{i p}-P_{i j}+v \partial_{p}^{2} A_{i j} .
$$

The advection term $-u_{m} \partial_{m} A_{i j}$ should now be taken into account in the evaluation of $F$ and $G$. Doing so, ignoring all the other terms, we have

$$
\left\langle\dot{A}_{i j} \dot{A}_{i j}\right\rangle_{S}=\left\langle u_{m} \partial_{m} A_{i j} u_{n} \partial_{n} A_{i j}\right\rangle,
$$

with the index $S$ denoting sweeping. We assume that the sweeping velocity and the velocity gradients are roughly independent (see for instance Refs. 28 and 29), so that

$$
\left\langle\dot{A}_{i j} \dot{A}_{i j}\right\rangle_{S}=\left\langle u_{m} u_{n}\right\rangle\left\langle\partial_{m} A_{i j} \partial_{n} A_{i j}\right\rangle .
$$

Invoking isotropy for the large-scale sweeping, this relation becomes

$$
\left\langle\dot{A}_{i j} \dot{A}_{i j}\right\rangle_{S}=\frac{1}{3}\left\langle u_{m} u_{m}\right\rangle\left\langle\partial_{n} A_{i j} \partial_{n} A_{i j}\right\rangle .
$$

This term can be expressed as a function of the energy spectrum. Assuming Kolmogorov scaling for this spectrum,

$$
E(k)=v^{5 / 4} \epsilon^{1 / 4} f_{u}(k \eta)
$$

we find that

$$
F_{S} \equiv\left\langle\dot{A}_{i j} \dot{A}_{i j}\right\rangle_{S}\left(\frac{v}{\epsilon}\right)^{2}=\frac{2}{3} \frac{\left\langle u_{m} u_{m}\right\rangle}{(\epsilon v)^{1 / 2}} \int \zeta^{4} f(\zeta) d \zeta,
$$

where $C_{\zeta}=\int \zeta^{4} f(\zeta) d \zeta$ is supposed to be of order unity. We similarly find $G_{S}=0$. If we substitute this in the Taylor expansion, we find that

$$
\tilde{B}_{1212}\left(t_{0}, t\right)=\tilde{B}_{1122}\left(t_{0}, t\right)=\tilde{B}_{1221}\left(t_{0}, t\right) \approx 1-\frac{1}{3} C_{\zeta}\left(\frac{t-t_{0}}{\tau_{K}}\right)^{2} \frac{\left\langle u_{m} u_{m}\right\rangle}{(\epsilon v)^{1 / 2}} .
$$

This shows that sweeping should decorrelate all correlations on the same time scale, and that this decorrelation is not independent of Reynolds number when scaled by Kolmogorov time-units. If one would like to collapse the different time-correlations, one should scale them by time units $\mathcal{T}=\tau_{K} R_{\lambda}^{-1 / 2}$. In Fig. 3 we show that this scaling allows to collapse the data better than the scaling by Kolmogorov-units. For high Reynolds numbers this decorrelation should therefore be much faster and the other terms, which were observed to lead to decorrelations over several Kolmogorov time scales, are therefore most probably negligible.

In order to obtain some more intuition on the dynamics, introducing the influence of the flow topology, we will now discuss simple heuristic models for the Lagrangian dynamics. 

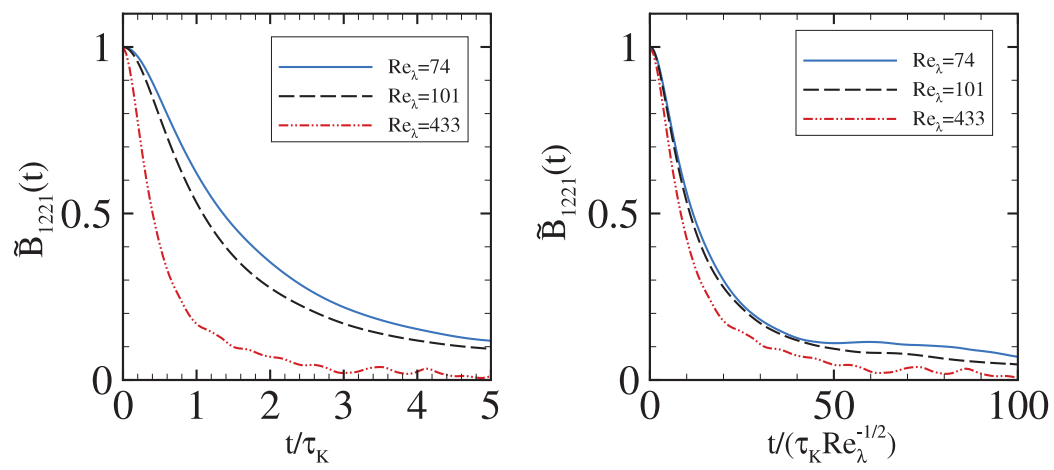

FIG. 3. Single-point two-time correlation of the gradient cross correlation $\tilde{B}_{1212}(t)$ at different Reynolds numbers, scaled by Kolmogorov time-units (left) and sweeping time-units (right).

\section{HEURISTIC MODELS FOR THE LAGRANGIAN VELOCITY GRADIENT CORRELATIONS}

\section{A. Strain and vorticity correlations}

The velocity gradient tensor can be decomposed without loss of generality into its rotation and strain part. This so-called Helmholtz decomposition (HD) leads to

$$
A_{i j}=S_{i j}+\Omega_{i j}
$$

with $S_{i j}=\left(\partial_{j} u_{i}+\partial_{i} u_{j}\right) / 2$ and $\Omega_{i j}=\left(\partial_{j} u_{i}-\partial_{i} u_{j}\right) / 2$. If we assume that both contributions remain independent of each other over the considered time-interval, we have

$$
\begin{aligned}
B_{i j m n}(t) & =\left\langle\left(S_{i j}(0)+\Omega_{i j}(0)\right)\left(S_{m n}(t)+\Omega_{m n}(t)\right)\right\rangle \\
& \approx\left\langle S_{i j}(0) S_{m n}(t)\right\rangle+\left\langle\Omega_{i j}(0) \Omega_{m n}(t)\right\rangle .
\end{aligned}
$$

In several recent studies of Lagrangian turbulence, it was observed that the time-correlations of strain and rotation could be approximated by exponentially decaying correlation functions. ${ }^{30-32}$ The two quantities are however correlated over different time scales. The characteristic time scales of shear and rotation are, respectively, about $2.3 \tau_{K}$ and $7.2 \tau_{K}$ with $\tau_{K}$ the Kolmogorov time scale. Combining this information with expression (28), we find

$$
\begin{aligned}
& \tilde{B}_{1212}(t)=\frac{3}{8} \exp \left(-\frac{t}{2.3 \tau_{K}}\right)+\frac{5}{8} \exp \left(-\frac{t}{7.2 \tau_{K}}\right) \\
& \tilde{B}_{1221}(t)=-\frac{3}{2} \exp \left(-\frac{t}{2.3 \tau_{K}}\right)+\frac{5}{2} \exp \left(-\frac{t}{7.2 \tau_{K}}\right) .
\end{aligned}
$$

These expressions, termed as the HD model, are compared to the DNS data, as shown in Fig. 4. It shows that the HD model captures the main characteristics for the Lagrangian correlations of the velocity gradients. However, the HD model does not reproduce the correct peak value of $\tilde{B}_{1221}$.

An additional insight is obtained by considering the two-dimensional case. In this case the vorticity is purely advected,

$$
\dot{\omega}=v \Delta \omega .
$$

On a Lagrangian trajectory, the vorticity is thus only decorrelated by viscous diffusion of vorticity. We write the vorticity as a function of the velocity gradients, $\omega=\left(A_{21}-A_{12}\right)$, so that the Lagrangian autocorrelation writes

$$
B_{\omega}(t)=\frac{\left\langle\omega\left(t_{0}\right) \omega(t)\right\rangle}{\left\langle\omega\left(t_{0}\right) \omega\left(t_{0}\right)\right\rangle}=2 \frac{\left\langle A_{12}\left(t_{0}\right) A_{12}(t)\right\rangle-\left\langle A_{12}\left(t_{0}\right) A_{21}(t)\right\rangle}{\left\langle\omega\left(t_{0}\right) \omega\left(t_{0}\right)\right\rangle} .
$$

Since in two dimensions $\left\langle A_{12}\left(t_{0}\right) A_{21}\left(t_{0}\right)\right\rangle=-\left\langle A_{12}\left(t_{0}\right) A_{12}\left(t_{0}\right)\right\rangle / 3$, we find that

$$
B_{\omega}(t)=\frac{3}{4} \tilde{B}_{1212}(t)+\frac{1}{4} \tilde{B}_{1221}(t) .
$$




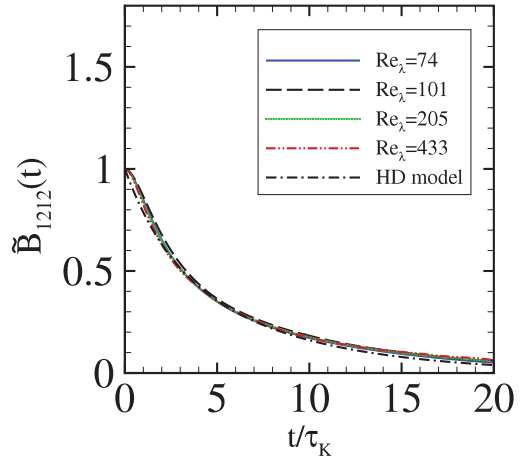

(a)

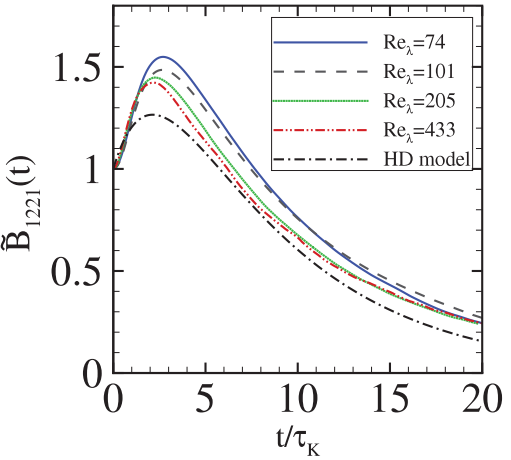

(b)

FIG. 4. Comparison between the HD model with DNS data. With increasing Reynolds numbers, the cross correlations $\tilde{B}_{1221}(t)$ from DNS data gradually approach the HD model. (a) $\tilde{B}_{1212}(t)$, (b) $\tilde{B}_{1221}(t)$.

If we neglect the viscous diffusion, the vorticity will not decorrelate on a trajectory. In this case $B_{\omega}(t)=1$ and we find

$$
\tilde{B}_{1221}(t)=4-3 \tilde{B}_{1212}(t) .
$$

This shows that if the autocorrelation $\tilde{B}_{1212}(t)$ decreases, say, as $\tilde{B}_{1212}(t)=1-\left(\left(t-t_{0}\right) / \tau\right)^{2}$, the cross correlation will increase as

$$
\tilde{B}_{1221}(t)=1+3\left(\left(t-t_{0}\right) / \tau\right)^{2} .
$$

In two dimensions the increase of the correlation $\tilde{B}_{1221}(t)$ can thus be understood by the inviscid mechanism of vorticity advection. The longer correlation time of the vorticity as compared to strain, in three dimensions could be reminiscent of this mechanism, even though in three dimensions the vorticity is not conserved due to the presence of vortex stretching. The investigation of the Lagrangian time-correlations of the velocity-gradient in two-dimensional turbulence is left for further research.

\section{B. Lagrangian correlations by assuming locally constant velocity gradients}

We will here try to identify the different effects that lead to the observed results. Therefore we will consider a given velocity field with locally constant velocity gradients. Subsequently we will show how, following a fluid particle on a trajectory, rotation and shear influence the Lagrangian correlations. Subsequently we add damping and as a last feature we allow the initially considered velocity field to decorrelate itself in time. This step by step complexification allows to disentangle the different physical mechanisms leading to the observations in the DNS.

\section{Stretching and rotating in an inviscid field}

We here introduce a simple heuristic model, where we can analytically compute the correlations. Our starting point is a velocity gradient field which we assume to be locally uniform and constant. In that case, locally the field can be considered as a 2D state (cf., Section 2.3.2 of Ref. 33). We choose the coordinate-system such that the velocity gradient is in the $x, y$-plane, with its axes chosen such that

$$
\boldsymbol{A}=\left[\begin{array}{lll}
-S & -\Omega & 0 \\
\Omega & S & 0 \\
0 & 0 & 0
\end{array}\right],
$$

with $S$ the pure irrotational strain rate and $\Omega$ the angular rotation rate. The associated flow-field is obviously not isotropic, but we will consider that the whole space is filled with an infinite number of 
local structures, and for each structure the characteristic orientations of the velocity gradient tensor are randomly distributed. The averages over all orientations will yield us isotropic statistics.

A rotation in a 3D space can be expressed using the rotation-transform tensor,

$\boldsymbol{Q}_{E}^{(\theta, \beta, \gamma)}=\left[\begin{array}{lll}\cos \theta \cos \gamma-\cos \beta \sin \theta \sin \gamma & \sin \theta \cos \gamma+\cos \beta \cos \theta \sin \gamma & \sin \beta \sin \gamma \\ -\cos \theta \sin \gamma-\cos \beta \sin \theta \cos \gamma & -\sin \theta \sin \gamma+\cos \beta \cos \theta \cos \gamma & \sin \beta \cos \gamma \\ \sin \beta \sin \theta & -\sin \beta \cos \theta & \cos \beta\end{array}\right]$,

where $\theta, \beta$, and $\gamma$ are a group of Euler angles in the directions of $z, x$, and $z$, respectively. In particular, when $\beta=\gamma=0$, it yields a rotation in the $x, y$-plane with angle $\theta$,

$$
\boldsymbol{Q}^{(\theta)}=\left[\begin{array}{lll}
\cos \theta & \sin \theta & 0 \\
-\sin \theta & \cos \theta & 0 \\
0 & 0 & 1
\end{array}\right]
$$

Rotating a tensor in $3 \mathrm{D}$ yields the velocity gradient

$$
\boldsymbol{A}^{(\theta, \beta, \gamma)}=\boldsymbol{Q}_{E}^{(\theta, \beta, \gamma)^{T}} \boldsymbol{A} \boldsymbol{Q}_{E}^{(\theta, \beta, \gamma)},
$$

with the superscripts $(\theta, \beta, \gamma)$ denoting the rotation angle, and $T$ the transposition of the matrix. This yields the off-diagonal components of the velocity gradient tensor $A_{12}^{(\theta, \beta, \gamma)}$ and $A_{21}^{(\theta, \beta, \gamma)}$.

The single-time velocity-gradient correlations $B_{1212}$ and $B_{1221}$ over an infinite number of randomly oriented realizations can then be calculated using the Haar measure of Euler angles,

$$
\begin{aligned}
& B_{1212} \equiv\left\langle A_{12} A_{12}\right\rangle=\frac{1}{8 \pi^{2}} \int_{0}^{2 \pi} d \theta \int_{0}^{\pi} \sin \beta d \beta \int_{0}^{2 \pi} d \gamma A_{12}^{(\theta, \beta, \gamma)} A_{12}^{(\theta, \beta, \gamma)}=\frac{1}{15}\left(3 S^{2}+5 \Omega^{2}\right), \\
& B_{1221} \equiv\left\langle A_{12} A_{21}\right\rangle=\frac{1}{8 \pi^{2}} \int_{0}^{2 \pi} d \theta \int_{0}^{\pi} \sin \beta d \beta \int_{0}^{2 \pi} d \gamma A_{12}^{(\theta, \beta, \gamma)} A_{21}^{(\theta, \beta, \gamma)}=\frac{1}{15}\left(3 S^{2}-5 \Omega^{2}\right) .
\end{aligned}
$$

In three dimensions, Eq. (7) shows that $B_{1221}=-B_{1212} / 4$. Combining this with Eq. (39), we find that $S^{2}=\Omega^{2}, B_{1212}=8 S^{2} / 15$, and $B_{1221}=-2 S^{2} / 15$. Comparing to Eq. (7), we also have $S^{2}=\epsilon /(4 v)$ which is in agreement with Eq. (35) and the definition of the dissipation rate.

We will now compute the evolution of the Lagrangian velocity-gradient correlations. In our homogeneous velocity-gradient field, we follow a fluid particle and we will determine how the velocity gradient is modified through the interaction of strain and rotation. In Section III, it was shown that the pressure Hessian might be an important factor for the short-time phenomenon of Lagrangian correlation; thus, an appropriate two-time model should consider the pressure Hessian. As it is difficult to rigorously take account of the pressure effect, we borrow the assumption of "frozen velocity gradient field" by Chevillard et al. ${ }^{15,34}$ Under this assumption, the deformation of a fluid particle is driven by a constant velocity gradient field during a short time of the order $\tau_{K}$. This deformation, on the one hand, implies the effect of pressure Hessian, ${ }^{15}$ on the other hand unfreezes, and changes the velocity gradient tensor. Specifically, for the present model, when an appropriate coordinate system is chosen, from Eq. (35), the influence of this frozen velocity gradient field is divided into the effects of stretching and rotation.

Considering the stretching effect by the uniform strain field, applying the strain in matrix Eq. (35) on a velocity gradient matrix $\boldsymbol{A}$ with a given time $t$ and shear rate $S$ leads to

$$
\boldsymbol{A}^{S}(t)=\boldsymbol{A}\left[\begin{array}{lll}
e^{-S t} & 0 & 0 \\
0 & e^{S t} & 0 \\
0 & 0 & 1
\end{array}\right]
$$

Then, by rotating this matrix over $\phi=\Omega t$, the gradient tensor is

$$
\boldsymbol{A}^{S,(\phi)}(t)=\boldsymbol{Q}^{(\phi)^{T}} \boldsymbol{A}^{S}(t) \boldsymbol{Q}^{(\phi)} .
$$

Note that here the rotation is always in the $x, y$-plane, and the transform matrix $\boldsymbol{Q}$, rather than $\boldsymbol{Q}_{E}$, should be used. Considering the random orientations, we have 


$$
\boldsymbol{A}^{S,(\phi),(\theta, \beta, \gamma)}(t)=\boldsymbol{Q}_{E}^{(\theta, \beta, \gamma)^{T}} \boldsymbol{Q}^{(\phi)^{T}} \boldsymbol{A}^{S}(t) \boldsymbol{Q}^{(\phi)} \boldsymbol{Q}_{E}^{(\theta, \beta, \gamma)},
$$

and the Lagrangian two-time correlation $B_{1212}(t)$ and $B_{1221}(t)$ are then

$$
\begin{aligned}
& B_{1212}(t)=\frac{1}{8 \pi^{2}} \int_{0}^{2 \pi} d \theta \int_{0}^{\pi} \sin \beta d \beta \int_{0}^{2 \pi} d \gamma A_{12}^{(\theta, \beta, \gamma)} A_{12}^{S,(\phi),(\theta, \beta, \gamma)}, \\
& B_{1221}(t)=\frac{1}{8 \pi^{2}} \int_{0}^{2 \pi} d \theta \int_{0}^{\pi} \sin \beta d \beta \int_{0}^{2 \pi} d \gamma A_{12}^{(\theta, \beta, \gamma)} A_{21}^{S,(\phi),(\theta, \beta, \gamma)} .
\end{aligned}
$$

From Eqs. (43) and (39), the normalized values can be obtained as

$$
\begin{aligned}
& \tilde{B}_{1212}(t) \equiv \frac{B_{1212}(t)}{B_{1212}(0)}=\frac{1}{3 S^{2}+5 \Omega^{2}}\left(\left(5 \Omega^{2}+3 S^{2} \cos (2 \phi)\right) \cosh (S t)-3 S \Omega \sin (2 \phi) \sinh (S t)\right), \\
& \tilde{B}_{1221}(t) \equiv \frac{B_{1221}(t)}{B_{1212}(0)}=\frac{1}{5 \Omega^{2}-3 S^{2}}\left(\left(5 \Omega^{2}-3 S^{2} \cos (2 \phi)\right) \cosh (S t)+3 S \Omega \sin (2 \phi) \sinh (S t)\right) .
\end{aligned}
$$

Using the isotropic result $S=\Omega$, these correlations can be expressed as a function of $\Omega$ alone. If we make the link with a turbulent flow, the dominant rotation time scale will be of the order of the Kolmogorov time scale. We therefore define $\Omega^{-1}$ for normalization with $\Omega^{-1} \sim \tau_{K}$. The resulting two-time correlation functions are shown in Fig. 5. It is shown that at short time, $\tilde{B}_{1212}(t)$ decreases while $\tilde{B}_{1221}(t)$ increases. Also the product of these two correlation functions, shown in Fig. 5(c), increases. Indeed, the phenomenological picture that we obtain from these considerations is the following: a fluid particle that is moving in vortical motion will change its orientation. The rotation of the local velocity gradient will then reorient so that after a typical small scale turn-over time, the velocity-gradient $\partial_{1} u_{2}$ will have changed towards a $\partial_{2} u_{1}$ local gradient. Apparently, this correlation increases from its initial value if we consider a given velocity-gradient field. At long times, all correlations attain nonphysically high values. Indeed, at long-times, the correlations are expected to decrease due to turbulent and viscous diffusion. We will therefore add a damping to the present model.

\section{Adding a damping function to the correlations}

In order to improve the temporal behaviour of the velocity-gradient correlation model, we add a damping function

$$
\begin{aligned}
& \tilde{B}_{1212}(t)=f(t)\left(\frac{1}{3 S^{2}+5 \Omega^{2}}\left(\left(5 \Omega^{2}+3 S^{2} \cos (2 \phi)\right) \cosh (S t)-3 S \Omega \sin (2 \phi) \sinh (S t)\right)\right), \\
& \tilde{B}_{1221}(t)=f(t)\left(\frac{1}{5 \Omega^{2}-3 S^{2}}\left(\left(5 \Omega^{2}-3 S^{2} \cos (2 \phi)\right) \cosh (S t)+3 S \Omega \sin (2 \phi) \sinh (S t)\right)\right) .
\end{aligned}
$$

Traditional damping models usually assume linear dissipation, ${ }^{19}$ which is probably a good approximation for $t \gg \tau_{K}$. However, we have seen that rigorously, for $t \ll \tau_{K}$ (expression (18)), the damping should be a quadratically decaying function of time. We have tried both types of damping. For

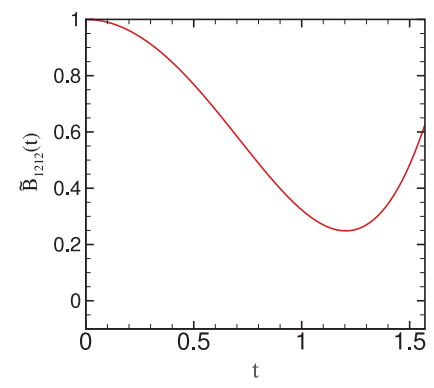

(a)

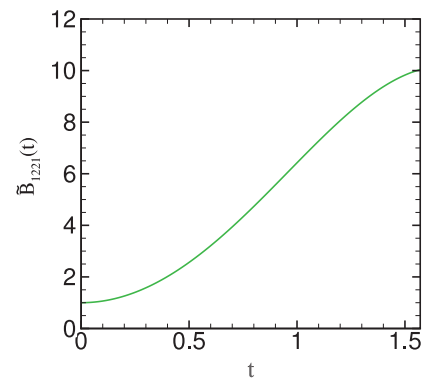

(b)

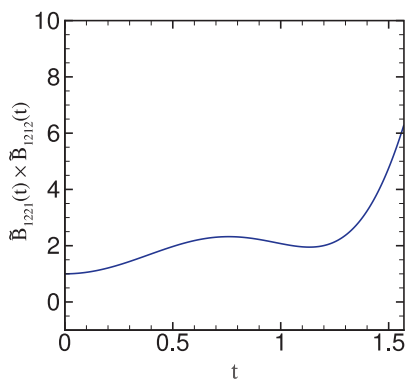

(c)

FIG. 5. The two-time correlation functions in a uniform stationary velocity gradient field without damping. (a) $\tilde{B}_{1212}(t)$, (b) $\tilde{B}_{1221}(t)$, (c) their product. 


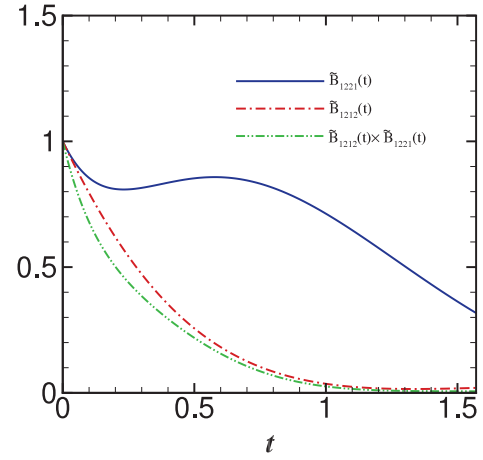

(a)

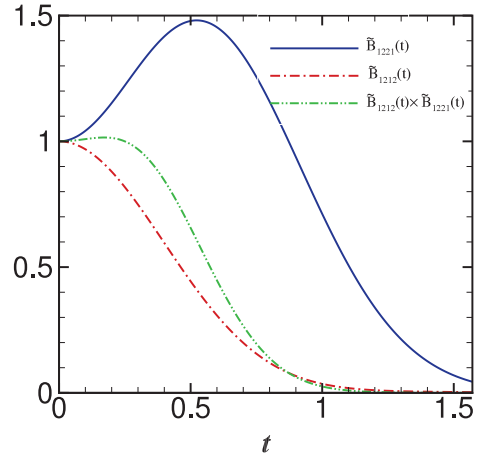

(b)

FIG. 6. The two-time correlation functions with damping. dash-dotted line: $\tilde{B}_{1212}(t)$; solid line: $\tilde{B}_{1221}(t)$; dash-dot-dotted line: their product. (a) Linear damping function; (b) quadratic damping function.

the linear damping, we use $f(t)=\exp \left(-c_{1} \Omega t\right)$ and for the quadratic damping $f(t)=\exp \left(-c_{2}(\Omega t)^{2}\right)$, with $c_{1}=c_{2}=2.2$, a constant which is arbitrarily chosen, but the value of which not qualitatively changes the behaviour as long as it is of order unity.

It is shown in Fig. 6 that the additional damping term allows the correlations to tend to zero for long times, as expected. However, the initial increase of the cross correlation is only observed in the case of a quadratic damping. The linear damping is incompatible with this feature. The peak time is related to the characteristic time of the damping model (cf. Refs. 20 and 35). This shows that if models for the Lagrangian evolution of the velocity-gradient tensor are to produce the short time statistics correctly, a quadratic damping should be used. Indeed, comparing to DNS results, for longer times the quadratic damping is over dissipative and the linear damping might prove to be more physically adapted. Hence, an interpolation between the two behaviours should be used in practice. Another possibility is to introduce more sophisticated damping models where the damping-time scale is not constant but evolves over its Lagrangian trajectory. ${ }^{25}$ We have instead chosen to improve the damping by evolving the quantities $S$ and $\Omega$ in our phenomenological model, using the time scales discussed in Sec. IV A.

\section{Damping the correlations using multiple time scales}

An alternative to the ad hoc damping function $f(t)$ is to allow the velocity gradients to decorrelate in inviscid model (44), taking into account that $S_{i j}$ and $\Omega_{i j}$ are governed by different time scales (as in Section IV A and discussed in Refs. 30-32). Additional coefficients are also required to relax the assumption of a frozen velocity gradient field and will be explained later. The multi-time scale kinetic model then writes

$$
\begin{gathered}
\tilde{B}_{1212}(t)=\frac{1}{3 S(0)^{2}+5 \Omega(0)^{2}}\left(\left(5 \Omega(t)^{2}+3 S(t)^{2} \cos \left(2 t \Omega_{r}(t)\right)\right) \cosh \left(t S_{r}(t)\right)\right. \\
\left.-3 S(t) \Omega(t) \sin \left(2 t \Omega_{r}(t)\right) \sinh \left(t S_{r}(t)\right)\right), \\
\tilde{B}_{1221}(t)=\frac{1}{5 \Omega(0)^{2}-3 S(0)^{2}}\left(\left(5 \Omega(t)^{2}-3 S(t)^{2} \cos \left(2 t \Omega_{r}(t)\right)\right) \cosh \left(t S_{r}(t)\right)\right. \\
\left.+3 S(t) \Omega(t) \sin \left(2 t \Omega_{r}(t)\right) \sinh \left(t S_{r}(t)\right)\right),
\end{gathered}
$$

where $S(t)=S(0) \exp \left(-t /\left(4.6 \tau_{K}\right)\right), \quad \Omega(t)=\Omega(0) \exp \left(-t /\left(14.4 \tau_{K}\right)\right), \quad S_{r}(t)=S\left(c_{r} t\right), \quad$ and $\quad \Omega_{r}(t)$ $=\Omega\left(c_{r} t\right)$. The coefficient $c_{r}$ is the relaxation of the assumption of frozen velocity gradient field, which allows different damping rates between the transform field and the defrozen quantity field. In practice, if we choose $c_{r}=9.0$, a good agreement with DNS results can be obtained (see Fig. 7). We remark here that the assumption of a frozen velocity gradient field by Chevillard et al. ${ }^{15,34}$ does not mean a constant field but implies a delay effect between the transform and the defrozen quantity field. According to Chevillard et al., this delay is physical and corresponds to the pressure redistribution 


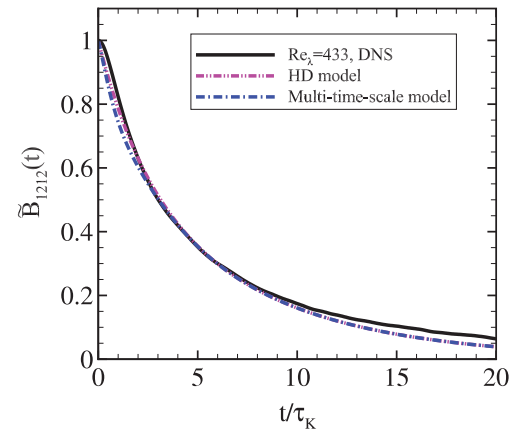

(a)

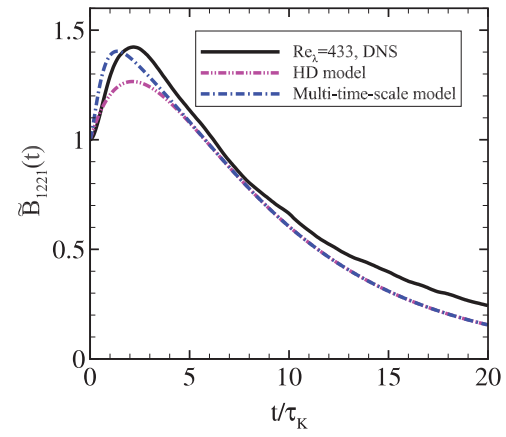

(b)

FIG. 7. Comparison between the multi-time scale kinetic model with HD model and DNS data, where $c_{r}=9.0$ in the multi-time scale kinetic model. (a) $\tilde{B}_{1212}(t)$, (b) $\tilde{B}_{1221}(t)$.

effects. In the multi-time scale model, the coefficient $c_{r}$ thus relaxes the freezing assumption and quantitatively describes this phenomenon of delay.

\section{Assessment of the models and comparison to DNS}

We have thus reproduced the observations in the simulations using heuristic models. The agreement of the multi-time scale model with DNS is better than that of the Helmholtz-decomposition assuming exponential time-correlations for strain and vorticity. However, the level of sophistication of the multi-time scale model is significantly higher and we have added an extra model-constant, characterizing the different damping rates between the transform field and the defrozen quantity field. Rather than a practical model, the multi-time scale model should be considered as a way to disentangle the different effects. Indeed, it shows that the initial tendencies of the velocity-gradient correlations can be reproduced by the combined effects of translation and rotation. At long times either a Gaussian/exponential damping function should be added to the correlation or the vorticity and strain along the trajectory should be taken to be decreasing functions in time. Finally, to optimize the agreement with DNS, the initially frozen velocity field should be defrozen by introducing a coefficient of relaxation. This model extends the studies of Chevillard et al. ${ }^{15,34}$ and proposes a relaxation of the assumption of a frozen velocity field.

\section{DISCUSSION AND CONCLUSION}

The fact observed in this paper, i.e., both $\tilde{B}_{1221}(t)$ and $\tilde{B}_{1212}(t) \tilde{B}_{1221}(t)$ always increase at short times, is surprising because these kinds of correlations are usually decaying in dissipative systems. We have tried to understand the short-time evolution of these correlations using kinematical considerations. In Section III, we have attempted to obtain rigorous results for the short-time correlations. It was shown, without even considering the Navier-Stokes equations, that the short-time evolution of the Lagrangian velocity gradient correlations is entirely determined by two invariants, $F \sim\left\langle\dot{A}_{i j} \dot{A}_{i j}\right\rangle$ and $G \sim\left\langle\dot{A}_{i j} \dot{A}_{j i}\right\rangle$. An increase of the Lagrangian cross correlation at short times should be observed if the ratio of the two invariants lies in the interval $1 / 4 \leq G / F \leq 4$. Subsequently we showed that the influence of viscosity gives $G / F=0$, thereby damping all correlations, as expected. However, symmetry arguments show that if we only consider the pressure Hessian, we find $G / F=1$, which should give rise to an increase of the cross correlation. We measured the ratio and found that $G / F \approx 0.6$ so that the initial evolution cannot be explained uniquely by the influence of the pressure Hessian. Also the influence of damping and self-interaction should be considered to understand the full picture.

Considering the Lagrangian time-correlation of the vorticity, it was shown that in the twodimensional inviscid case, the increases of $\tilde{B}_{1212}(t)$ and $\tilde{B}_{1221}(t)$ are kinematically coupled and an increase of one of the correlations implies the decorrelation of the other. The presence of vortex 
stretching in three dimensions does not allow such a simple conclusion, but if the vorticity correlation is more persistent than the $\tilde{B}_{1212}(t)$ correlation, an initial increase of $\tilde{B}_{1221}(t)$ is expected. By contrast, the presence of sweeping in the Eulerian framework does not allow to observe any increase of time-correlations.

To give a more intuitive understanding of the link between flow-topology and Lagrangian evolution, two heuristic models were proposed. The Helmholtz-decomposition model can qualitatively reproduce the observed effect. Then, we aim at disentangling the different physical mechanisms leading to the observations in the DNS, which finally lead to the multi-time scale model validated by the quantitative agreement with DNS. These models, on the one hand, explain the physical roles of stretching, rotation, pressure, and damping in the present observation, on the other hand, show limitations of the traditional linear damping at short times and support a multiple-time scale damping to relax the assumption of a frozen velocity gradient field.

\section{ACKNOWLEDGMENTS}

This work is supported by the 973 program of China (No. 2013CB834100), the National Natural Science Foundation of China (Nos. 11202013, 11572025, 51420105008, 11232011, and 11472277), and the National Natural Science Associate Foundation (NSAF) of China (Grant No. U1230126). G.D.J. benefitted from the hospitality of the Nordic Institute for Theoretical Physics under the auspices of the program "Dynamics of Particles in Flows: Fundamentals and Applications" in June 2014 in Sweden. The anonymous referees are much appreciated for the constructive suggestions to improve the manuscript.

${ }^{1}$ C. Meneveau, "Lagrangian dynamics and models of the velocity gradient tensor in turbulent flows," Annu. Rev. Fluid Mech. 43, 219-245 (2011).

2 J. M. Wallace and P. V. Vukoslavcevic, "Measurement of the velocity gradient tensor in turbulent flows," Annu. Rev. Fluid Mech. 42, 157-181 (2010).

${ }^{3}$ M. Guala, A. Liberzon, A. Tsinober, and W. Kinzelbach, "An experimental investigation on Lagrangian correlations of small-scale turbulence at low Reynolds number," J. Fluid Mech. 574, 405-427 (2007).

${ }^{4}$ Wm. T. Ashurst, A. R. Kerstein, R. M. Kerr, and C. H. Gibson, "Alignment of vorticity and scalar gradient with strain rate in simulated Navier-Stokes turbulence," Phys. Fluids 30(8), 2343-2353 (1987).

${ }^{5}$ T. Ishihara, Y. Kaneda, M. Yokokawa, K. Itakura, and A. Uno, "Small-scale statistics in high-resolution direct numerical simulation of turbulence: Reynolds number dependence of one-point velocity gradient statistics," J. Fluid Mech. 592, 335-366 (2007).

${ }^{6}$ B. J. Cantwell, "Exact solution of a restricted Euler equation for the velocity gradient tensor," Phys. Fluids 4, 782 (1992).

${ }^{7}$ B. J. Cantwell, "On the behavior of velocity gradient tensor invariants in direct numerical simulations of turbulence," Phys Fluids 5, 2008-2013 (1993).

${ }^{8}$ J. Martin, A. Ooi, M. S. Chong, and J. Soria, "Dynamics of the velocity gradient tensor invariants in isotropic turbulence," Phys. Fluids 10, 2336-2346 (1998).

${ }^{9}$ Y. Li and C. Meneveau, "On the origin of non-Gaussian statistics in hydrodynamic turbulence," Phys. Rev. Lett. 96, 164502 (2005).

${ }^{10}$ B. J. Cantwell, "A singularity-free model of the local velocity gradient and acceleration gradient structure of turbulent flow," in Tubes, Sheets and Singularities in Fluid Dynamics, Proceedings of the IUTAM Symposium, edited by K. Bajer and H. K. Moffatt (Kluwer Academic Publishers, New York, 2002), pp. 247-260.

${ }^{11}$ A. Ooi, J. Martin, J. Soria, and M. S. Chong, "A study of the evolution and characteristics of the invariants of the velocity-gradient tensor in isotropic turbulence," J. Fluid Mech. 381, 141-174 (1999).

${ }^{12}$ B. Luthi, M. Holzner, and A. Tsinober, "Expanding the QR space to three dimensions," J. Fluid Mech. 641, 497-507 (2009).

${ }^{13}$ M. Chertkov, A. Pumir, and B. I. Shraiman, "Lagrangian tetrad dynamics and the phenomenology of turbulence," Phys. Fluids 11(8), 2394-2410 (1999).

${ }^{14}$ A. Naso and A. Pumir, "Scale dependence of the coarse-grained velocity derivative tensor structure in turbulence," Phys. Rev. E 72(5), 056318 (2005).

${ }^{15}$ L. Chevillard, C. Meneveau, L. Biferale, and F. Toschi, "Modeling the pressure Hessian and viscous Laplacian in turbulence: Comparisons with direct numerical simulation and implications on velocity gradient dynamics," Phys. Fluids 20, 101504 (2008).

${ }^{16} \mathrm{M}$. Wilczek and C. Meneveau, "Pressure Hessian and viscous contributions to velocity gradient statistics based on Gaussian random fields," J. Fluid Mech. 756, 191-225 (2014).

${ }^{17}$ A. Pumir, E. Bodenschatz, and H. T. Xu, "Tetrahedron deformation and alignment of perceived vorticity and strain in a turbulent flow," Phys. Fluids 25(3), 035101 (2013).

${ }^{18}$ L. Chevillard and C. Meneveau, "Lagrangian time correlations of vorticity alignments in isotropic turbulence: Observations and model predictions," Phys. Fluids 23(10), 101704 (2011).

19 J. Martin, C. Dopazo, and L. Valino, "Dynamics of velocity gradient invariants in turbulence: Restricted Euler and linear diffusion models," Phys. Fluids 10, 2012-2025 (1998). 
${ }^{20} \mathrm{M}$. Martins-Afonso and C. Meneveau, "Recent fluid deformation closure for velocity gradient tensor dynamics in turbulence: Timescale effects and expansions," Physica D 239, 1241-1250 (2010).

${ }^{21}$ H. D. Yu and C. Meneveau, "Lagrangian refined Kolmogorov similarity hypothesis for gradient time-evolution in turbulent flows," Phys. Rev. Lett. 104, 084502 (2010).

${ }^{22}$ H. D. Yu and C. Meneveau, "Scaling of conditional Lagrangian time correlation functions of velocity and pressure gradient magnitudes in isotropic turbulence," Flow, Turbul. Combust. 85(3-4), 457-472 (2010).

${ }^{23}$ G. D. Jin and G. W. He, "A nonlinear model for the subgrid timescale experienced by heavy particles in large eddy simulation of isotropic turbulence with a stochastic differential equation," New J. Phys. 15, 035011 (2013).

${ }^{24}$ G. W. He, G. D. Jin, and X. Zhao, "Scale-similarity model for Lagrangian time correlations in isotropic and stationary turbulence," Phys. Rev. E 80(6), 066313 (2009).

${ }^{25}$ E. Jeong and S. S. Girimaji, "Velocity-gradient dynamics in turbulence: Effect of viscosity and forcing," Theor. Comput. Fluid Dyn. 16, 421-432 (2003).

${ }^{26}$ Y. Li, E. Perlman, M. Wan, Y. Yang, R. Burns, C. Meneveau, S. Chen, A. Szalay, and G. Eyink, "A public turbulence database cluster and applications to study Lagrangian evolution of velocity increments in turbulence," J. Turbul. 9, N31 (2008).

${ }^{27}$ Y. Yang, G. W. He, and L. P. Wang, "Effects of subgrid-scale modeling on Lagrangian statistics in large-eddy simulation," J. Turbul. 9, N8 (2008).

${ }^{28}$ G. W. He and J. B. Zhang, "Elliptic model for space-time correlation in turbulent shear flows," Phys. Rev. E 73(5), 055303 (2006).

${ }^{29}$ X. Zhao and G. W. He, "Space-time correlations of fluctuating velocities in turbulent shear flows," Phys. Rev. E 79(4), 046316 (2009).

${ }^{30}$ M. Shin and D. L. Koch, "Rotational and translational dispersion of fibres in isotropic turbulent flows," J. Fluid Mech. 540, 143-173 (2005).

${ }^{31}$ D. Vincenzi, S. Jin, E. Bodenschatz, and L. R. Collins, "Stretching of polymers in isotropic turbulence: A statistical closure," Phys. Rev. Lett. 98, 024503 (2007).

32 A. Pumir and M. Wilkinson, “Orientation statistics of small particles in turbulence,” New J. Phys. 13, 093030 (2011).

${ }^{33}$ P. Sagaut and C. Cambon, Homogeneous Turbulence Dynamics (Cambridge University Press, 2008).

${ }^{34}$ L. Chevillard and C. Meneveau, "Lagrangian dynamics and statistical geometric structure of turbulence," Phys. Rev. Lett. 97, 174501 (2006).

35 J. Martin, A. Ooi, C. Dopazo, M. S. Chong, and J. Soria, "The inverse diffusion time scale of velocity gradients in homogeneous isotropic turbulence," Phys. Fluids 9(4), 814 (1997). 